\title{
Centrifugal Microfluidic Control Mechanisms for Biosensors
}

\author{
Masaki Yamaguchi* and Vivek Shetty ${ }^{1}$ \\ Department of Mechanical Engineering \& Robotics, Graduate School of Science \& Technology, \\ Shinshu University, 3-15-1 Tokida, Ueda, Nagano 386-8567, Japan \\ ${ }^{1}$ Section of Oral and Maxillofacial Surgery, 23-009 UCLA Health Science Center, \\ 10833 Le Conte Avenue, Los Angeles, CA 90095-1668, USA
}

(Received February 8, 2016; accepted April 1, 2016)

Keywords: centrifugal microfluidics, immunosensor, flow channel, fluid valve, lab-on-a-CD

Compact biosensors have been recognized as being useful for analyzing biomarkers such as protein and gene products from the micro- or nano-liter scale of human samples for diagnosis of human disease, and have led to the development of lab-on-a-chip or micro-total-analysis systems. Biosensor platforms allow near real-time detection and estimation of human disease indicators. Following a brief introduction of three elementary functions of biosensors - the molecular recognition element, the transducer element, and the microfluidic control mechanism-this review focuses on the current status of development of microfluidic control mechanisms for biosensors, and future technological advances. In this review, we highlight the recent experimental advances in centrifuge-based microfluidic platforms (centrifugal microfluidics), which aim to increase a biosensor's sensitivity and selectivity. Particular focus is placed on the application of hydrophobic micropatterns to centrifugal microfluidics. Centrifugal microfluidics will render biomarkers more field-practical and accessible to end-users in a cost-effective manner.

\section{Introduction}

A quarter of century has passed since a mediator-type glucose enzyme sensor was put on the market for medical use. ${ }^{(1-3)}$ Presently, near-patient glucose monitoring for the management of diabetes is well-established and biosensors are a dominant part of the home testing market. ${ }^{(4,5)}$ Building on this work, numerous biosensors have been introduced for the diagnosis of diseases, health monitoring, food safety, and environmental monitoring. ${ }^{(6)}$ The growing demand for compact biosensor devices for analyzing human samples such as proteins, mRNA, and DNA on the micro- or nano-liter scale has led to the development of the concept of lab-on-a-chip (LOC) ${ }^{(7)}$ and micro-totalanalysis systems ( $\mu$ TAS). ${ }^{(8)}$ Point-of-care testing (POCT) is particularly valued for its portability, immediate availability of test results, and multiple monitoring capabilities.

Most biosensors have three essential components: a molecular recognition element interacting with the target analyte; a transducer element; and a microfluidic control mechanism translating the biorecognition event into a useful electrical signal. The transducer element serves as the signal amplifier in many cases. To improve the performance of biosensors, microfluidic control mechanisms are the key technology concepts. ${ }^{(9)}$ There are various techniques for moving microliter volumes of fluids within biosensor devices. The use of centrifuge-based microfluidic platforms

"Corresponding author: e-mail: masakiy@shinshu-u.ac.jp 
(centrifugal microfluidics) is one effective way to manipulate the flow of biofluids and reagents for analysis in the micro-flow channels of a biosensor. ${ }^{(10,11)}$

This paper reviews the centrifugal microfluidic control mechanisms of biosensors based on papers published in scholarly journals. Particular focus is placed on the application of hydrophobic micropatterns to centrifugal microfluidics, which will lead to increasing implementation in diagnostic biosensors.

\section{Microfluidic Propulsion Techniques}

\subsection{Scale effect in microfluidic control mechanisms}

Micro-electromechanical systems (MEMS) allow the fabrication of various types of microfluidic control mechanisms which may be applied to biosensors. ${ }^{(12)}$ The microfluidic control mechanism commonly has flow channels connecting reservoirs. The volume of sample solution correlates with the time required to diffuse over a reservoir. The diffusional effects are expressed according to Brownian motion and the Stokes-Einstein equation. The diffusion coefficient, $D\left(\mathrm{~m}^{2} / \mathrm{s}\right)$, is proportional to the square of the random-walk step size, $\delta$ (diffusion length in $\mathrm{m}$ ). ${ }^{(13)}$

$$
\delta=\sqrt{2 D \tau},
$$

where $\tau$ is the time (s) required for a molecule to diffuse over distance $\delta$. It takes $500 \mathrm{~s}$ to diffuse over a distance of $1 \mathrm{~mm}$. If the distance is reduced by an order of magnitude, the time is reduced by two orders of magnitude (Fig. 1)..$^{(13-15)}$ Miniaturizing the microfluidic control mechanism might be

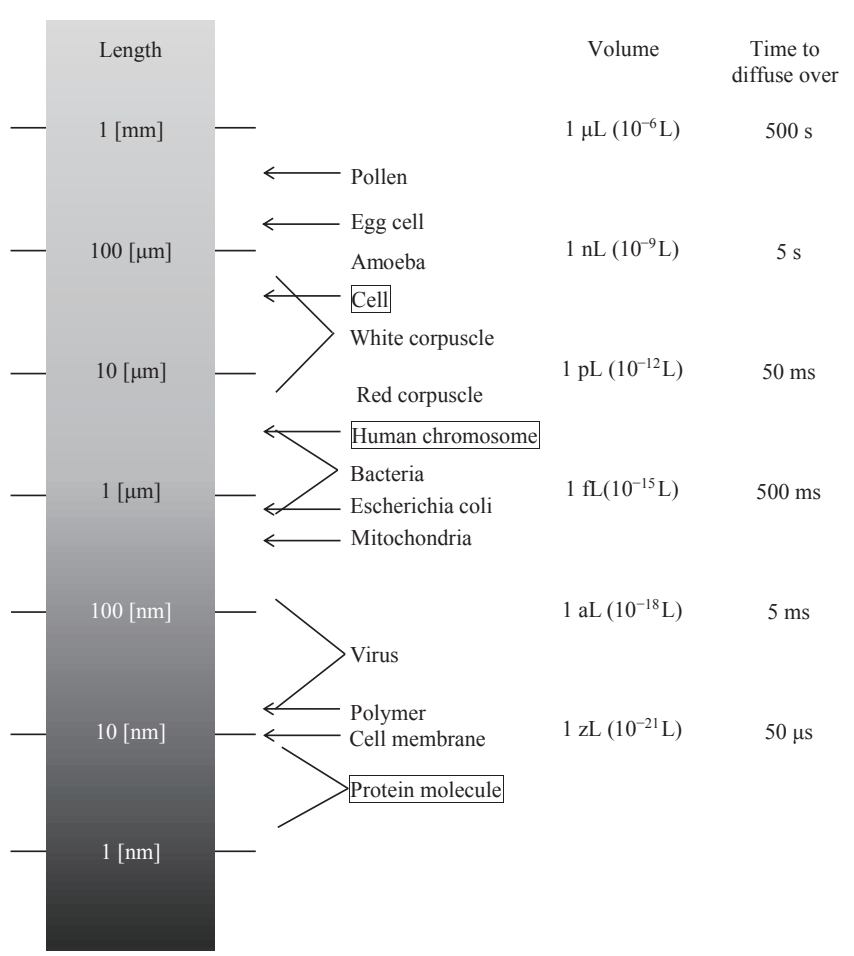

Fig. 1. Calculated parameter sets for diffusion of a sample solution in a microfluidic control mechanism. 
an effective strategy for shortening the time to complete the analysis in a biosensor. To analyze one molecule of a biochemical, the size of the reservoir may be reduced to $100 \mathrm{~nm}$. However, a $1 \mathrm{fL}$ or $1 \mathrm{aL}$ of water can evaporate easily at room temperature. Therefore, to accomplish rapid analysis of biochemicals, miniaturized microfluidic propulsion techniques are required.

\subsection{Microfluidic propulsion techniques}

There are various techniques for moving micro- and nano-liter volumes of fluids, with commonly used microfluidic propulsion techniques including capillary force, centrifugal force, pressure, heat driving force, ultrasonic driving force, and electric-field driving force (Table 1). ${ }^{(10)}$ These microfluidic propulsion techniques are summarized and compared as follows:

A. Capillary force: This phenomenon is caused by surface tension, and the fluid velocity increases as the cross section of the flow channel decreases. The flow speed can be controlled by the density and pore rate of the material such as cellulose or plastic resin. The lack of need for an energy supply is a strong point and renders capillary force suitable for developing low-cost and disposable sensors. ${ }^{(16)}$

B. Centrifugal force: Compact disc (CD)-based microfluidics is most popular when it comes to adapting centrifugal forces to a microfluidic propulsion technique. ${ }^{(11)}$ These techniques are being used increasingly in the development of the concept of lab-on-a-CD. ${ }^{(17)}$ A centrifugal fluid valve can be established by a flow channel merged to a reservoir. When the centrifugal fluid valve rotates, both surface tension and centrifugal force are exerted on the fluid. When the centrifugal force exceeds the surface tension, the valve opens. The angular acceleration can be controlled precisely using a stepping motor, so this method does not require developing a specific actuator. Today, centrifugal microfluidics has evolved into a mature technology in the areas of chemical analysis for industrial use and the medical diagnosis of diseases.

C. Pressure: Propulsion techniques have been utilized to move a liquid by transforming the wall of a reservoir into a piezoelectric element made of lead zirconate titanate (PZT). ${ }^{(18)}$ The weakness of this method is that a comparatively large sized element is necessary compared to the quantity of displacement. Long-distance propulsion by pressure is challenging because fluid resistance becomes extremely large at the micrometer scale.

D. Heat-drive: Joule heat generated by a heating element causes the rapid vaporization of liquid in a chamber and forms a bubble, which causes a large pressure increase that can propel a droplet of liquid. ${ }^{(19)}$ This technique is called thermal drop-on-demand in the printing field, and most consumer inkjet printers adopt this method; but it may not be suitable for propulsion biomarkers such as labile proteins that are easily denatured by heat.

E. Acoustic drive: Acoustic droplet ejection is also common in this area. ${ }^{(20)}$ A localized acoustic vibration transmitted on a solid surface is called a surface acoustic wave (SAW). The SAW damps the emission of a longitudinal wave in a liquid under the condition that a liquid exists on the propagation face. When the amplitude of SAW exceeds the threshold level, the liquid vibrates, flows, or flies from the surface. A set of interdigitated electrodes on the surface of a PZT are needed to form a SAW device. ${ }^{(21)}$ A SAW device can be used for propulsion not only of liquids but also of powders.

F. Electric-field driving force: This technique includes electrophoresis, ${ }^{(22)}$ electro-osmosis, ${ }^{(23)}$ and electro-wetting. ${ }^{24)}$ Electrophoresis is based on the principle that Coulomb force acts on ions in 
Table 1

Driving force principles for microfluidic propulsion techniques.

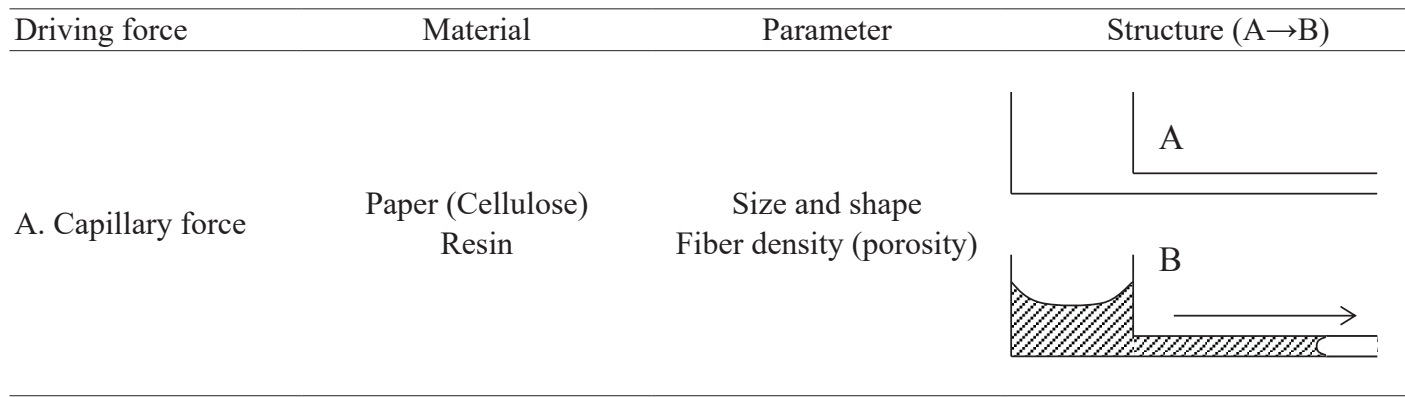

$\begin{array}{llc} & \text { Resin } & \text { Size and shape } \\ \text { B. Centrifugal force } & \text { Glass } & \text { Acceleration }\end{array}$

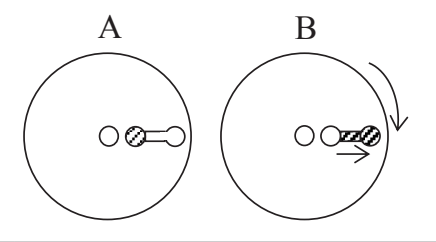

$\begin{array}{cccc}\text { Resin } & \text { Size and shape } & \text { Frequency } \\ \text { C. Pressure } & \text { Amplitude of voltage } & \text { A } & \end{array}$

$\begin{array}{llll}\text { D. Heat-drive } & \begin{array}{c}\text { Resin } \\ \text { Metal }\end{array} & \begin{array}{c}\text { Size and shape } \\ \text { Temperature }\end{array} \\ \text { E. Acoustic drive } & \begin{array}{c}\text { Size and shape } \\ \text { Frequency } \\ \text { Amplitude of voltage }\end{array} & \text { PZT }\end{array}$

\begin{tabular}{|c|c|c|c|c|}
\hline \multirow{4}{*}{ F. Electric field drive } & \multirow{4}{*}{$\begin{array}{c}\text { Resin } \\
\text { Glass } \\
\text { Metal electrode }\end{array}$} & \multirow{4}{*}{$\begin{array}{c}\text { Size and shape } \\
\text { Voltage } \\
\text { pH }\end{array}$} & $\begin{array}{l}\mathrm{A} \\
\mathrm{O}\end{array}$ & $\begin{array}{r}\text { B } \\
\text { O } \\
\end{array}$ \\
\hline & & & 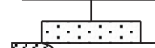 & $\frac{1}{r \cdots \cdots}$ \\
\hline & & & $\mathscr{W}$ & 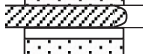 \\
\hline & & & $\because \because \cdots$ & \\
\hline & & & 0 & 0 \\
\hline
\end{tabular}

PZT: lead zirconate titanate

liquids in an electric field. Electro-osmosis is caused by the induced Coulomb force, which acts when an electric double layer is formed. Electro-wetting produces a fluid valve by controlling the wettability of a solid surface (which is typically hydrophobic) in an applied electric field. These techniques have the advantage of low power consumption. 


\section{Measurement Techniques of Biosensors}

\subsection{Molecular recognition element}

First, we summarize the underlying principles of molecular recognition elements used for the detection of biomarkers. ${ }^{(25)}$ The complexities and specificities of the biosensing process increase in the following order: enzymatic methods, antigen-antibody methods, and hybridization methods; and the cost of the tests increases correspondingly. The increasing availability of enzyme-linked immunosorbent assay (ELISA) kits has led to a surge of interest in biomarkers. Gold colloid-based methods now allow for low-cost qualitative tests manifesting color reactions as a visible line on a biochip. Similarly, enzyme sensors, immunosensors, and surface plasmon resonance sensors(26) display the color reactions of the ELISA process by way of electrochemical or optical phenomena and thus allow measurements that are both highly sensitive and continuous. A nanoscale optical biosensor based on localized surface plasmon resonance (LSPR) spectroscopy has been developed to monitor the interactions between antigens and antibodies. ${ }^{(27)}$ The DNA chip (DNA microarray) is a tool used to identify mutations in genes. The DNA chip, which consists of a small glass plate encased in plastic, is manufactured in a manner similar to a computer microchip. The surface of each chip can contain thousands of short, synthetic, single-stranded DNA sequences. When the chip is exposed to a biofluid, competitive hybridization occurs between the immobilized synthetic cDNA/cRNA and mRNA/DNA strands in a sample. Fluorescence tagging makes automatic detection possible. Because chip technology is still relatively new, it is currently only used as a research tool.

\subsection{Transducer element}

Commonly used transducer elements are optical, mechanical, or electrical-based, for generating light, current, or frequency signals (Table 2). Depending on the nature of the recognition event, the biosensing platform might be either a bioaffinity or a biocatalytic platform. Bioaffinity devices rely on the selective binding of the target analyte to a surface-confined capture element (e.g., antibody, oligonucleotide). Biocatalytic devices, in contrast, use an immobilized enzyme for recognizing the target substrate. When exposed to a biofluid such as a saliva sample, the interaction of the analyte with the bioreceptor produces a detectable effect that is measured by the transducer and converted into a measurable output such as an electrical signal. The strength of the electrical signal is proportional to the level of a single analyte or group of analytes, and the result is presented on an

Table 2

Detection methods used for transducer elements.

\begin{tabular}{lc}
\hline Optical & Absorbance-based \\
& Visible light \\
& Fluorescence \\
& Luminescence \\
& Imaging \\
\hline Mechanical & Mass-sensitive element \\
\hline Electrical & Electrochemical-based \\
& Electrokinetic-based \\
\hline
\end{tabular}


optical display. Depending on the biomarker of interest, the biosensing platform can utilize antigen/ antibody binding, nucleic acid interactions, or enzymatic interactions to recognize the analyte. Common forms of transducers tend to use optical detection (luminescence, absorption, surface plasmon resonance, and others).

\section{Centrifugal Microfluidics}

\subsection{Theory of the centrifugal fluid valve}

In the case of centrifugal microfluidics, there are two forces: the surface tension that derives from the material, and the centrifugal force generated by rotation exerted on the fluid. (28) The centrifugal fluid valve opens when the equilibrium between the two forces is broken. The pressure depends on the surface tension, $\Delta P_{\mathrm{S}}$, and is expressed as follows: ${ }^{(29,30)}$

$$
\Delta P_{\mathrm{s}}=\frac{2\left(\gamma_{\mathrm{SV}}-\gamma_{\mathrm{SL}}\right)}{d}
$$

where $d$ is capillary radius when a flow channel is approximated by a cylindrical tube, and $\gamma_{\mathrm{SV}}$ and $\gamma_{\mathrm{SL}}$ are the surface tensions of the solid-air and solid-liquid, respectively.

The pressure depends on the centrifugal force $\Delta P_{\mathrm{c}}$ and is expressed in terms of the difference in diameter at each end of the flow channel, $\Delta r$, as follows:

$$
\Delta P_{\mathrm{c}}=\rho \omega^{2} \bar{r} \Delta r
$$

where $\rho$ is fluid density, $\omega$ is the angular frequency, and $\bar{r}$ is the average distance of the liquid in the flow channel from the center of rotation. The force when a liquid pass through a flow channel, $\Delta F$, is given by the difference between centrifugal force and surface tension.

\subsection{Centrifugal disc-chip}

Centrifugal microfluidics is a mature technology, and multiple-sensor devices have been proposed based on it. ${ }^{(31)}$ An automated immunosensor, a type of lab-on-a-CD, incorporates two centrifugal fluid valves on a disposable CD-based chip that allows for rapid reporting of hormone levels. ${ }^{(32)}$ A CD-based chip with a diameter of $120 \mathrm{~mm}$ was fabricated using an acrylic resin, and an incorporated centrifugal fluid valve provides the fluid control mechanism [Fig. 2(a)]. The disposable CD-based chip is comprised of the following surface elements: a buffer reservoir, an injection reservoir, a substrate reservoir, a reaction reservoir, and a waste fluid reservoir, all of which are interconnected by miniature flow channels. The flow channels function as valves. A polystyrene pad integrated into the disc-chip is sealed with a transparent upper sealing layer [Fig. 2(b)]. The immunosensor system comprises a disposable CD-based chip, a portable optical reader equipped with an amplifier, a stepping motor with a control unit, and a laptop computer that controls the component processes [Fig. 2(c)].

Figure 3(a) describes the mode of operation for the centrifugal fluid valves which control the flow of the biofluid. Figure 3(b) shows the principle of the immunoreaction underlying the molecular recognition of cortisol. This system adopts a competitive reaction using an alkaline 


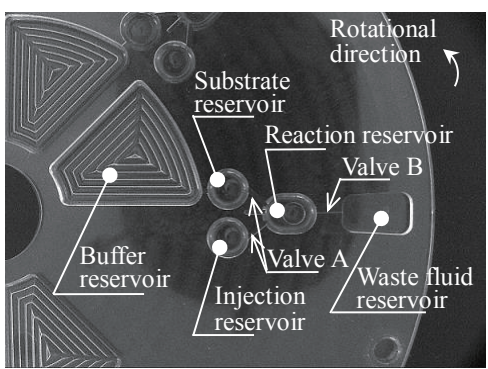

(a)

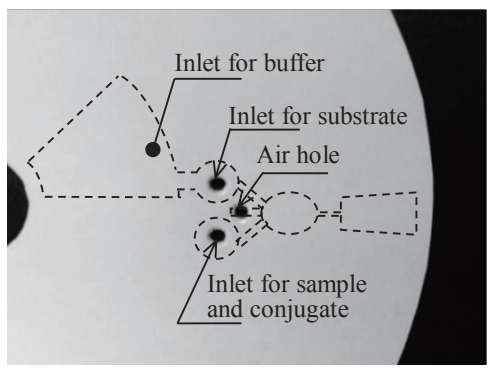

(b)

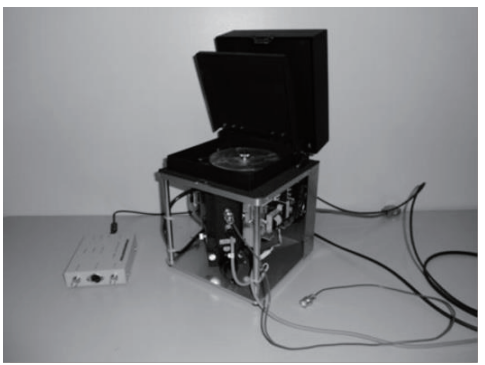

(c)

Fig. 2. Immunosensor mounted with a centrifugal fluid control mechanism. ${ }^{(32)}$ (a) Disc-chip, (b) upper sealing layer of disc-chip, and (c) fabricated monitor (Yamaguchi, et al., Sens. BioSens. Res. 2014).

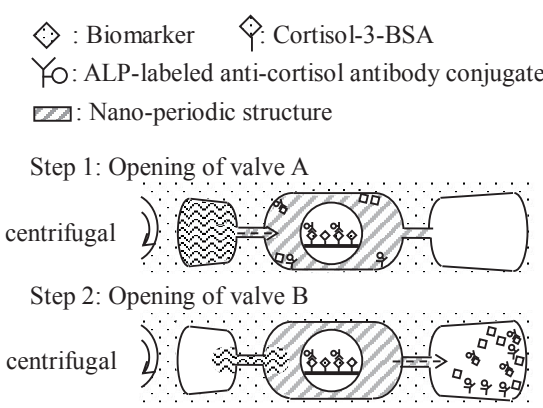

(a)

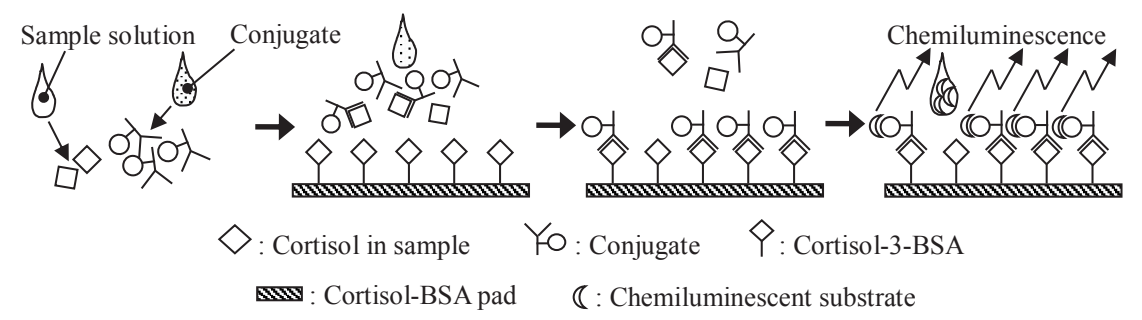

(b)

Fig. 3. (a) A set of centrifugal fluid valves and the chemical reaction of the immunosensor. (b) Immunoreaction principle underlying the molecular recognition of biomarkers such as cortisol (Yamaguchi, et al., Sens. BioSens. Res. 2014).

phosphatase (ALP)-labeled anti-cortisol antibody conjugate. Similar to this system, the fluid control mechanisms are commonly fabricated on the surfaces of polymer, glass, or silicon, with polymers receiving particular attention in recent years. ${ }^{(33)}$ The water repellency of polymers is sometimes insufficient to control the fluids and to prevent non-specific binding of proteins in the sample solution. Surface modification, by applying a surfactant or a hydrophobic coating agent, is a common and effective method for preventing non-specific binding. Thus, the improvement of hydrophilicity or hydrophobicity can increase the biosensor's sensitivity and selectivity. ${ }^{(34,35)}$ However, the downside is that the chemically treated agents can dissolve in the sample solution and influence the results of the chemical reaction. 


\subsection{Application of hydrophobic micropatterns}

In many fields of technology, numerous studies on surface science have expanded our understanding of the repellency of liquid drops on a surface. ${ }^{(36)}$ One of the ways of controlling the wettability of a solid surface is to increase the surface roughness. Investigators have attempted to improve the physical wettability of solid surfaces by introducing a periodic structure on the surface. ${ }^{(37)}$ The lotus leaf is symbolic of an extremely hydrophobic surface characterized by a high contact angle. ${ }^{(38)}$ Superhydrophobic surfaces with a high contact angle above $150^{\circ}$ exhibit extreme water repellence and self-cleaning properties. ${ }^{(39)}$ Gao and colleagues reported on the progress of surface roughnessinduced wettability throughout history. ${ }^{(40)}$

Surface roughness-induced wettability is prevalent in nature, and similar physical performance has been a driver to switch from "natural" design to industrial design. To improve the performance of surfaces, such as their self-cleaning properties, ${ }^{(41)}$ periodic structures have been applied to industrial and bioengineering products. ${ }^{(42)}$ Previously, there have been some reports of methods that chemically modify molecules on a surface. ${ }^{(43,44)}$ In particular, one effective approach to modifying the surface roughness-induced wettability is to use injection molded plastic resins, because the wettability of the surface of a plastic product can be controlled by adding a periodic structure to the surface of the mold.

To clarify its effectiveness, a periodic structure was applied to the fluid control mechanism on a plastic plate to improve its hydrophobicity. A CD-based chip with a centrifugal fluid valve was designed previously by the authors [Figs. 4(a) and 4(b)].(45) Micro-periodic structures were

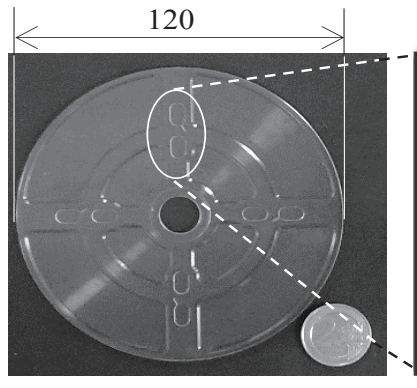

(a)

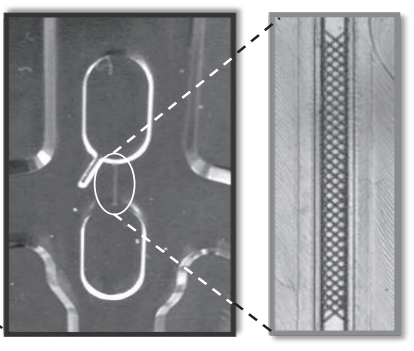

(b) (c)

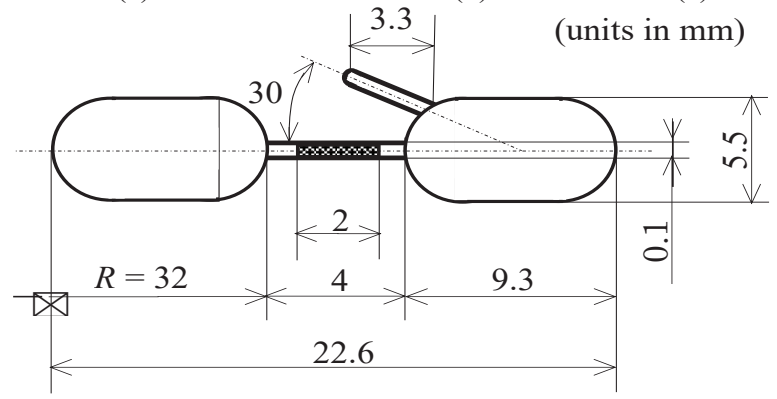

(d)

Fig. 4. (a) External view of a CD-based chip, (b) two reservoirs joined by flow channel, (c) flow channel with micro-periodic structure, and (d) a centrifugal fluid valve with a micro-periodic structure (Shimakura and Yamaguchi, J. Adhes. Sci. Technol. 2015). 


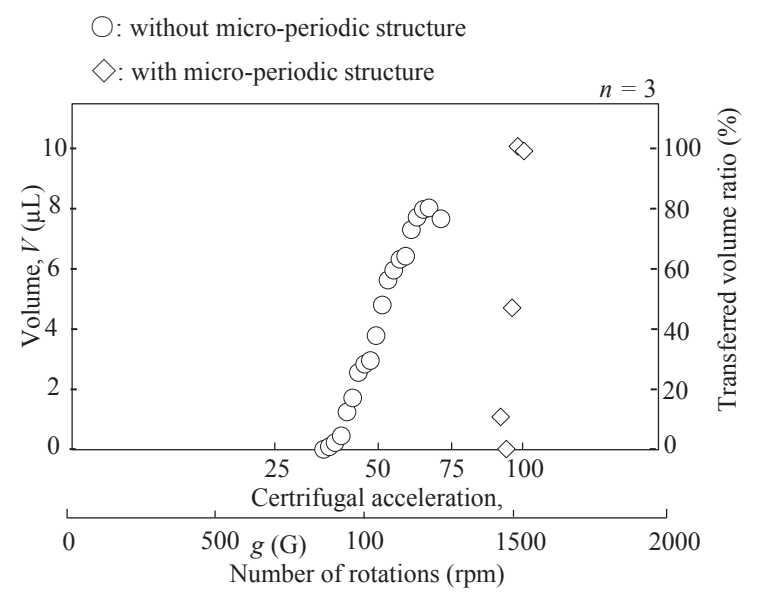

Fig. 5. Relationship between volume, $V$, and centrifugal acceleration, $g$, for the CD-based chip with centrifugal fluid valve ( $2 \mathrm{~g} / \mathrm{L}$ of protein solution).

impressed on the flow channel of the centrifugal fluid valve on the CD-based chip [Figs. 4(c) and 4(d)]. The apparent contact angles, $\theta^{\prime}$, and the contact angle hysteresis, $C A H$, are normally used to evaluate wettability. The $C A H$ is calculated from the difference between the advancing contact angle, $\theta_{\mathrm{a}}$, and the receding contact angle, $\theta_{\mathrm{r}}{ }^{(46)}$ The sessile drop technique was used, which is the most common technique for measuring $C A H \cdot{ }^{(47)}$ The $C A H$ directly characterizes resistance to mobility of a water droplet; low values confirm a lack of pinning, consistent with a nearly defectfree surface. ${ }^{(48,49)}$

The liquid transfer characteristics of the centrifugal fluid valve were evaluated by the transferred volume ratio of the sample solution, $V$, and the slope, $\Delta V_{\mathrm{r}}$. It is common to use concentrations of protein of 2 and $80 \mathrm{~g} / \mathrm{L}$ because they are similar to the total protein concentrations in saliva ${ }^{(50)}$ and plasma $^{(51)}(1-3 \mathrm{~g} / \mathrm{L})$ and serum $(60-80 \mathrm{~g} / \mathrm{L}) .^{(52,53)}$ The transferred volume ratio increased by $20 \%$ compared to the flow channel without a micro-periodic structure when a protein solution similar to the concentrations in saliva and plasma was used (Fig. 5). Similar to the results of transferred volume ratio, the parallel pillars improved the slope, meaning that a change in rotation velocity of only $49.3 \mathrm{rpm}$ was needed to activate the centrifugal fluid valve.

\section{Conclusion}

The rapidly evolving disciplines of proteomics, bioengineering, analytic chemistry, and computational biology are producing a variety of new tools and biosensing platforms that are wellequipped to monitor the presence or absence of an increasing array of biomarkers. Indeed, some have already been deployed in devices that are already used in clinical settings for the diagnosis and/or predicting the prognosis of certain diseases.

In particular, early work with automated biosensors involving centrifugal microfluidics suggests that there could be essential elements for portable biosensor platforms that provide for measurement of biomarkers within a shortened timespan compatible with point-of-care applications. Applying periodic structure to fluid control mechanisms is an effective means of realizing hydrophobic surfaces. 


\section{Acknowledgements}

This work was supported in part by Grant No. 25350517 from the Japan Society for the Promotion of Science, Proposal for Fluid Control Mechanism Based on the Wettability and Establishment of a Quantitative and Automated Biosensor (P.I.-M. Yamaguchi), Japan.

\section{References}

1 L. C. Clark and C. Lyons: Ann. N. Y. Acad. Sci. 102 (1962) 29.

2 D. R. Matthews, R. R. Holman, E. Bown, J. Steemson, A. Watson, and S. Hughes: The Lancet 4 (1987) 778.

3 B. A. Gregg and A. Heller: Anal. Chem. 62 (1990) 258.

4 J. D. Newman and A. P. F. Turner: Biosens. Bioelectron. 20 (2005) 2435.

5 L. Carver: IEEE Spectr. 2015 (2015) S24.

6 K. Toko ed.: Biochemical Sensors (Pan Stanford Publishing, Singapore, 2013) Parts 1-3.

7 P. Abgrall and A.-M. Gué: J. Micromech. Microeng. 17 (2007) R15.

8 G. Testa, G. Persichetti, and R. Bernini: Sensors 15 (2015) 465.

9 K. F. Lei: J. Lab. Autom. 17 (2012) 330.

10 M. Madou, J. Zoval, G. Jia, H. Kido, J. Kim, and N. Kim: Annu. Rev. Biomed. Eng. 8 (2006) 601.

11 O. Strohmeier, M. Keller, F. Schwemmer, S. Zehnle, D. Mark, F. von Stetten, R. Zengerle, and N. Paust: Chem. Soc. Rev. 44 (2015) 6187.

12 M. J. Madou ed.: Fundamentals of Microfabrication (CRC Press, New York, 1997) Chap. 7.

13 M. J. Madou ed.: Fundamentals of Microfabrication, The Science of Miniaturization, Second Edition (CRC Press, New York, 2002) Chap. 9.

14 A. Manz, D. J. Harrison, E. M. J. Verpoorte, J. C. Fettinger, A. Paulus, H. Ludi, and H. M. Widmer: J. Chromatogr., A 593 (1992) 253.

15 P. R. Brown and E. Grushka eds.: Advances in Chromatography (Marcel Dekker Inc., New York, 1993) Chap. 1.

16 M. Yamaguchi, Y. Matsuda, S. Sasaki, M. Sasaki, Y. Kadoma, Y. Imai, D. Niwa, and V. Shetty: Biosens. Bioelectron. 41 (2013) 186.

17 L. X. Kong, A. Perebikovsky, J. Moebius, L. Kulinsky, and M. Madou: J. Lab. Autom. 20 (2015) 1.

18 D. J. Laser and J. G. Santiago: J. Micromech. Microeng. 14 (2004) R35.

19 N. J. Nielsen: Hewlett-Packard J. 36 (1985) 4.

20 J. Olechno, C. Green, and L. Rasmussen: J. Lab. Autom. 21 (2016) 1.

21 S. Shiokawa and J. Kondoh: Electron. Commun. Jpn. 79 (1996) 42.

22 J. W. Jorgenson and E. J. Guthrie: J. Chromatography 255 (1983) 335.

23 J. Tamada, N. Bohannon, and R. Potts: Nat. Med. 1 (1995) 1198.

24 N. Nashida, W. Satoh, J. Fukuda, and H. Suzuki: Biosens. Bioelectron. 15 (2007) 3167.

25 M. Yamaguchi and V. Shetty: Electrochemistry 79 (2011) 442.

26 D. C. Cullen, R. G. Brown, and C. R. Lowe: Biosensors 3 (1987-1988) 211.

27 A. J. Haes and R. P. Van Duyne: J. Am. Chem. Soc. 124 (2002) 10596.

28 M. R. McNeely, M. K. Spute, N. A. Tusneem, and A. R. Oliphant: SPIE 3877 (1999) 210.

29 R. D. Johnson, I. H. A. Badr, G. Barrett, S. Lai, Y. Lu, M. J. Madou, and L. G. Bachas: Anal. Chem. 73 (2001) 3940.

30 D. D. Nolte: Rev. Sci. Instrum. 80 (2009) 101101-1.

31 R. Burger, L. Amato, and A. Boisen: Biosens. Bioelectron. 76 (2016) 54.

32 M. Yamaguchi, H. Katagata, Y. Tezuka, D. Niwa, and V. Shetty: Sens. Bio-Sens. Res. 1 (2014) 15.

33 A. Bange, H. B. Halsall, and W. R. Heineman: Biosens. Bioelectron. 20 (2005) 2488.

34 V. Biju: Chem. Soc. Rev. 43 (2014) 744.

35 E. S. Redeker, D. T. Ta, D. Cortens, B. Billen, W. Guedens, and P. Adriaensens: Bioconjugate Chem. 24 (2013) 1761.

36 B. H. P. Rosado and C. D. Holder: Ecohydrology 6 (2013) 150.

37 R. Fürstner, W. Barthlott, C. Neinhuis, and P. Walzel: Langmuir 21 (2005) 956.

38 A. Solga, Z. Cerman, B. F. Striffler, M. Spaeth, and W. Barthlott: Bioinspiration Biomimetics 2 (2007) S126.

39 M. Nosonovsky and B. Bhushan: Green Tribology (Springer-Verlag, Heidelberg, 2008) pp. 25-40.

40 L. Gao, T. J. McCarthy, and X. Zhang: Langmuir 25 (2009) 14100. 
41 H. Dodiuk, S. Kenig, and A. Dotan: J. Adhes. Sci. Technol. 26 (2012) 701.

42 E. Ueda and P. A. Levkin: Adv. Mater. 25 (2013) 1234.

43 B.-B. Wang, J.-T. Feng, Y.-P. Zhao, and T. X. Yu: J. Adhes. Sci. Technol. 24 (2010) 2693.

44 B.-B. Wang, J.-T. Feng, Y.-P. Zhao, and T. X. Yu: J. Adhes. Sci. Technol. 25 (2010) 93.

45 T. Shimakura and M. Yamaguchi: J. Adhes. Sci. Technol. 29 (2015) 2565.

46 E. Bormashenko: Science 291 (2013) 339.

47 L. Románszki, M. Mohos, J. Telegdi, Z. Keresztes, and L. Nyikos: Per. Pol. Chem. Eng. 58 (2014) 53.

48 M. Delmas, M. Monthioux, and T. Ondarcuhu: Phys. Rev. Lett. 106 (2011) 136102-1.

49 T.-S. Wong, S. H. Kang, S. K. Y. Tang, E. J. Smythe, B. D. Hatton, A. Grinthal, and J. Aizenberg: Nature 477 (2011) 443.

50 C. Dawes: J. Physiol. 320 (1981): 139.

51 A. Tollan, K. Kvenild, H. Strand, P. Øian, and J. M. Maltau: Contraception 45 (1992) 473.

52 A. J. Macleod and O. Drummond: Dev. Biol. Stand. 46 (1980) 17.

53 B. G. Blijenberg and B. Leijnse: J. Clin. Chem. Clin. Biochem. 21 (1983) 601. 